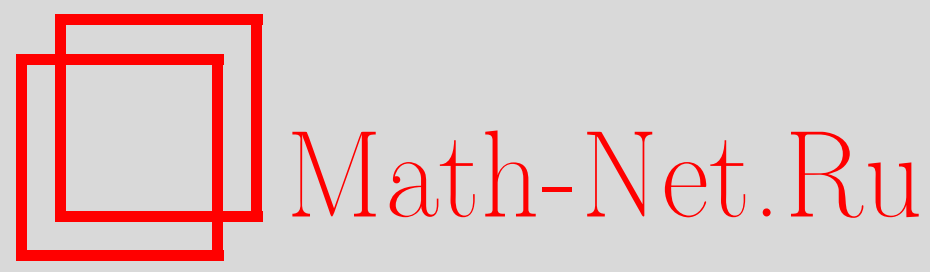

А. С. Белов, Применение комплексного анализа для оценки снизу тригонометрических полиномов, Матем. заметки, 1998, том 63, выпуск 6, 803-811

DOI: https://doi.org/10.4213/mzm1350

Использование Общероссийского математического портала Math-Net.Ru подразумевает, что вы прочитали и согласны с пользовательским соглашением http://www . mathnet.ru/rus/agreement

Параметры загрузки:

IP : 54.92 .164 .108

26 апреля 2023 г., 12:58:17

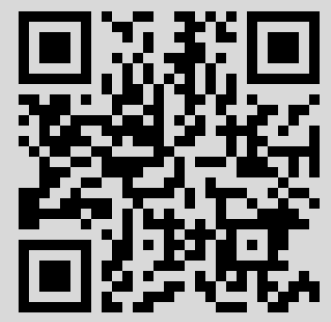




\section{ПРИМЕНЕНИЕ КОМПЛЕКСНОГО АНАЛИЗА ДЛЯ ОЦЕНКИ СНИЗУ ТРИГОНОМЕТРИЧЕСКИХ ПОЛИНОМОВ}

А. С. Белов

В работе доказано, что для любых различных натуральных чисел $k_{1}, \ldots, k_{n}$ и произвольных действительных чисел $a_{1}, \ldots, a_{n}$ справедливо неравенство

$$
-\min _{x} \sum_{j=1}^{n} a_{j}\left(\cos \left(k_{j} x\right)-\sin \left(k_{j} x\right)\right) \geqslant B\left(\frac{1}{1+\ln n} \sum_{j=1}^{n} a_{j}^{2}\right)^{1 / 2}, \quad n \in \mathbb{N},
$$

где $B$ - положительная абсолютная постоянная (например, $B=1 / 8$ ). Приведен пример, который показывает, что в этом неравенстве порядок относительно $n$, т.е. множитель $(1+\ln n)^{-1 / 2}$ нельзя улучшить. Получен также более изящный аналог неравенства Пихоридеса и некоторые другие оценки снизу тригонометрических сумм.

Библиограффия: 6 названий.

В работе [1] Пихоридес получил следующий результат.

Пусть $n$ - натуральное число, $k_{1}, \ldots, k_{n}$ - различные натуральные числа, $c_{1}, \ldots, c_{n}-$ произвольные комплексные числа и

$$
F(x)=\sum_{j=1}^{n} c_{j} e^{i k_{j} x}, \quad M_{F}=-\min _{x} \operatorname{Re} F(x) .
$$

Пусть $c_{1}^{*}, \ldots, c_{n}^{*}$ - соответственно числа $\left|c_{1}\right|, \ldots,\left|c_{n}\right|$, переставленные в невозрастающем порядке. Тогда при $M_{F}>0$ верно неравенство

$$
M_{F} \ln M_{F}+\|F\|_{1} \geqslant B \sum_{j=1}^{n} \frac{c_{j}^{*}}{j}-A .
$$

Здесь и далее, через $A, A_{1}, \ldots$ (через $B, B_{1}, \ldots$ ) обозначим достаточно большую (достаточно малую) положительную абсолютную постоянную, в каждом случае свою. Если $f \in L_{2 \pi}^{p}, p \in(0, \infty)$, то, как обьгно,

$$
\|f\|_{p}=\left(\frac{1}{2 \pi} \int_{0}^{2 \pi}|f(t)|^{p} d t\right)^{1 / p} .
$$

Работа выполнена при финансовой поддержке Российского фонда фундаментальных исследований, грант № 96-01-00094. 
Для любого положительного $\gamma$ неравенство (2) применим к полиному $\gamma F$. В результате, разделив обе части полученного неравенства на $\gamma$, имеем

$$
M_{F} \ln \left(\gamma M_{F}\right)+\frac{A}{\gamma}+\|F\|_{1} \geqslant B \sum_{j=1}^{n} \frac{c_{j}^{*}}{j} .
$$

Минимизируя по $\gamma$, т.е., полагая $\gamma=A / M_{F}$, приходим к неравенству

$$
(1+\ln A) M_{F}+\|F\|_{1} \geqslant B \sum_{j=1}^{n} \frac{c_{j}^{*}}{j} .
$$

Таким образом, справедливо неравенство

$$
M_{F}+\|F\|_{1} \geqslant B_{1} \sum_{j=1}^{n} \frac{c_{j}^{*}}{j} .
$$

Поскольку $1+M \ln M \geqslant M$ при $M>0$, из неравенства (3) сразу вытекает (2). Следовательно, неравенства (2) и (3) эквивалентны. Непосредственно из определения (1) вытекает, что всегда $M_{F} \geqslant 0$, причем $M_{F}=0$ тогда и только тогда, когда все коэффициенты $c_{j}=0, j=1, \ldots, n$. Поэтому неравенство (3) верно и при $M_{F}=0$.

Рассмотрим полином

$$
F(x)=2 \sum_{k=1}^{n}\left(1-\frac{k}{n+1}\right) e^{i k x} .
$$

В силу известных свойств ядра Фейера $M_{F} \leqslant 1$, а правая часть неравенства (3) имеет порядок $1+\ln n$, т.е. в левой части неравенства $(3)$ второе слагаемое, которое имеет порядок $1+\ln n$, нельзя отбросить. Следующий пример показывает, что нельзя отбросить и первое слагаемое:

$$
F(x)=\sum_{k=1}^{m}\left(1-\frac{k}{m+1}\right)\left(e^{i(m+1-k)}+e^{i(m+1+k)}\right),
$$

где $m$ - наибольшее натуральное число такое, что $2 m \leqslant n$.

В этой статье мы получим неравенство (3), как следствие следующей теоремы.

ТЕорема 1. Пусть функиия $\Phi \in H^{1}, \Phi(z)=\sum_{j=0}^{\infty} c_{j} z^{j}$ - ее разложсение в ряд Тейлора, $c_{0}$ - вещественное число и $\operatorname{Re} \Phi(z) \geqslant 0$ при $|z|<1$. Тогда верно неравенство

$$
A_{1}\|\Phi\|_{H^{1}} \geqslant \sum_{j=1}^{\infty} \frac{c_{j}^{*}}{j}
$$

əде $\left\{c_{j}^{*}\right\}_{j=1}^{\infty}-$ перестановка последовательности $\left\{\left|c_{j}\right|\right\}_{j=1}^{\infty}$ в невозрастаюшем порядке.

Если $p \in(0, \infty)$, то запись $\Phi \in H^{p}$, как обычно, означает, что функция $\Phi$ аналитична в круге $|z|<1$ и

$$
\|\Phi\|_{H^{p}}=\sup _{r \in[0,1)}\left(\frac{1}{2 \pi} \int_{0}^{2 \pi}\left|\Phi\left(r e^{i t}\right)\right|^{p} d t\right)^{1 / p}<\infty .
$$

Если в теореме $1 \Phi(z)=M_{F}+\sum_{j=1}^{n} c_{j} z^{k_{j}}$, то имеем 
СлЕДСТВИЕ 1. Для любых различных натуральных чисел $k_{1}, \ldots, k_{n}$, комплексных чисел $c_{1}, \ldots, c_{n}$ для полинома (1) справедливо неравенство (3) с постоянной $B_{1}=1 / A_{1}$.

Действительно, в этом случае гармоническая функция $\operatorname{Re} \Phi(z) \geqslant 0$ при $|z|=1$, а значит и при $|z|<1$, и $\|\Phi\|_{H^{1}}=\left\|\Phi\left(e^{i t}\right)\right\|_{1} \leqslant M_{F}+\|F\|_{1}$. Таким образом, неравенство (3) является простым следствием теоремы 1.

ДОКАЗАТЕЛЬСТВо ТЕОРЕМЫ 1 . Можно считать, что функция $\Phi$ не равна тождественно 0, иначе (4) очевидно. Не уменьшая общности считаем, что $\|\Phi\|_{H^{1}}=1$ (если необходимо, функцию $\Phi$ можно умножить на положительную постоянную). Поскольку $\operatorname{Re} \Phi(z) \geqslant 0$ при $|z|<1$, выделим аналитическую в круге $|z|<1$ ветвь функции $\ln (1+\Phi(z))=\ln |1+\Phi(z)|+i \varphi(z)$, где $|\varphi(z)|<\pi / 2, \varphi(0)=0$. Пусть $r \in[0,1)$, $f_{r}(t)=\operatorname{Re} \Phi\left(r e^{i t}\right), \tilde{f}_{r}(t)=\operatorname{Im} \Phi\left(r e^{i t}\right)$. Повторяя рассуждения, используемые при доказательстве известной теоремы М. Рисса (см. [2, т. 1, с. 404], [3, с. 571], [4, с. 127]), по теореме Коши имеем

$$
\frac{1}{2 \pi} \int_{0}^{2 \pi} \Phi\left(r e^{i t}\right) \ln \left(1+\Phi\left(r e^{i t}\right)\right) d t=\Phi(0) \ln (1+\Phi(0)) .
$$

Приравнивая действительные части, получим равенство

$$
\frac{1}{2 \pi} \int_{0}^{2 \pi} f_{r}(t) \ln \left|1+f_{r}(t)+i \widetilde{f}_{r}(t)\right| d t=\Phi(0) \ln (1+\Phi(0))+\frac{1}{2 \pi} \int_{0}^{2 \pi} \widetilde{f}_{r}(t) \varphi\left(r e^{i t}\right) d t,
$$

из которого непосредственно вытекает неравенство

$$
\frac{1}{2 \pi} \int_{0}^{2 \pi} f_{r}(t) \ln \left|1+f_{r}(t)\right| d t \leqslant \Phi(0) \ln (1+\Phi(0))+\frac{1}{4} \int_{0}^{2 \pi}\left|\widetilde{f}_{r}(t)\right| d t .
$$

Поскольку $\Phi(0) \leqslant\|\Phi\|_{H^{1}}=1,\left\|\widetilde{f}_{r}\right\|_{1} \leqslant\|\Phi\|_{H^{1}}=1$, из (5) следует оценка

$$
\frac{1}{2 \pi} \int_{0}^{2 \pi} f_{r}(t) \ln \left(1+f_{r}(t)\right) d t \leqslant \ln 2+\frac{\pi}{2} \text {. }
$$

Поэтому для неотрицательной функции

$$
f(t)=\lim _{r \rightarrow 1-0} f_{r}(t) \text { почти всюду }
$$

по лемме Фату получаем

$$
\frac{1}{2 \pi} \int_{0}^{2 \pi} f(t) \ln (1+f(t)) d t \leqslant \ln 2+\frac{\pi}{2}
$$

Поскольку коэффициенты $\Phi$ урье $\widehat{c}_{j}(f)=c_{j} / 2$ при $j \geqslant 1$ и для перестановки модулей коэффищиентов Фурье в невозрастающем порядке верна оценка (см. [2, т. 2, с. 236, задача 8$]$ )

то

$$
\sum_{j=1}^{\infty} \frac{c_{j}^{*}(f)}{j} \leqslant A_{2}+A_{3}\left\|f \ln ^{+} f\right\|_{1}
$$

$$
\frac{1}{2} \sum_{j=1}^{\infty} \frac{c_{j}^{*}}{j} \leqslant A_{2}+A_{3}\left(\ln 2+\frac{\pi}{2}\right) .
$$

Неравенство (4) доказано с постоянной $A_{1}=2 A_{2}+(\ln 4+\pi) A_{3}$. Применяя интерполящионную теорему Марцинкевича, несложно показать, что можно выбрать $A_{2}=6$, $A_{3}=8$. Поэтому в теореме 1 можно считать $A_{1}=60$.

Теорема 1, а значит, и следствие 1 доказаны.

Кроме того, из теоремы 1 вытекает 
СлЕДСТВИЕ 2. Пусть функиия $\Phi \in H^{1}, \Phi(z)=\sum_{j=0}^{\infty} c_{j} z^{j}-$ ее разложсение в ряд Тейлора, $\left\{c_{j}^{*}\right\}_{j=1}^{\infty}-$ перестановка последовательности $\left\{\left|c_{j}\right|\right\}_{j=1}^{\infty}$ в невозрастающем порядке и $\Phi^{*}(z)=c_{0}+\sum_{j=1}^{\infty} c_{j}^{*} z^{j}$. Тогда если $\operatorname{Re} \Phi(z) \geqslant 0$ при $|z|<1$, mо $\Phi^{*} \in H^{1}$ и справедливо неравенство

$$
A_{4}\|\Phi\|_{H^{1}} \geqslant\left\|\Phi^{*}\right\|_{H^{1}} .
$$

ДокАЗАТЕльство. Функция $\Phi_{0}(z)=\operatorname{Re} c_{0}+\sum_{j=1}^{\infty} c_{j} z^{j}$ удовлетворяет всем условиям теоремы 1. Поскольку $\left|\operatorname{Im} c_{0}\right| \leqslant\left|c_{0}\right| \leqslant\|\Phi\|_{H^{1}}$, то $\left\|\Phi_{0}\right\|_{H^{1}} \leqslant\left|\operatorname{Im} c_{0}\right|+\|\Phi\|_{H^{1}} \leqslant$ $2\|\Phi\|_{H^{1}}$. Применяя к функции $\Phi_{0}$ теорему 1 , получаем

$$
2 A_{1}\|\Phi\|_{H^{1}} \geqslant A_{1}\left\|\Phi_{0}\right\|_{H^{1}} \geqslant \sum_{j=1}^{\infty} \frac{c_{j}^{*}}{j} .
$$

Если $r \in[0,1)$ и $|t| \in(\pi /(n+1), \pi / n]$ при некотором натуральном $n$, то

$$
\begin{aligned}
\left|\Phi^{*}\left(r e^{i t}\right)\right| & \leqslant\left|c_{0}\right|+\sum_{j=1}^{n} c_{j}^{*} r^{j}+\left|\sum_{j=n+1}^{\infty} c_{j}^{*} r^{j} e^{i j t}\right| \\
& \leqslant\left|c_{0}\right|+\sum_{j=1}^{n} c_{j}^{*}+\frac{c_{n+1}^{*} r^{n+1}}{|\sin (t / 2)|} \leqslant\left|c_{0}\right|+\sum_{j=1}^{n} c_{j}^{*}+(n+1) c_{n+1}^{*} .
\end{aligned}
$$

Поэтому

$$
\begin{aligned}
\frac{1}{2 \pi} \int_{-\pi}^{\pi}\left|\Phi^{*}\left(r e^{i t}\right)\right| d t & \leqslant \frac{1}{2 \pi} \sum_{n=1}^{\infty} 2\left(\frac{\pi}{n}-\frac{\pi}{n+1}\right)\left(\left|c_{0}\right|+\sum_{j=1}^{n} c_{j}^{*}+(n+1) c_{n}^{*}\right) \\
& =\left|c_{0}\right|+2 \sum_{n=1}^{\infty} \frac{c_{n}^{*}}{n} \leqslant\left(1+4 A_{1}\right)\|\Phi\|_{H^{1}} .
\end{aligned}
$$

Отсюда получаем, что функция $\Phi^{*} \in H^{1}$ и верно неравенство $\left\|\Phi^{*}\right\|_{H^{1}} \leqslant\left(1+4 A_{1}\right)\|\Phi\|_{H^{1}}$.

Отметим, что согласно известному неравенству Харди (см. [2, т. 1, с. 454])

$$
\pi\left\|\Phi^{*}\right\|_{H^{1}} \geqslant\left|c_{0}\right|+\sum_{j=1}^{\infty} \frac{c_{j}^{*}}{j} .
$$

Поэтому следствие 2 эквивалентно теореме 1. Это следствие интересно сопоставить со следующей теоремой.

ТЕОРема 2. Мохно построить неотрицательную четную функиию $f \in L_{2 \pi}$ такую, что если

$$
\widehat{a}_{k}(f)=\frac{1}{\pi} \int_{0}^{2 \pi} f(t) \cos (k t) d t
$$

- ее действительные коэффичиенты Фурье, $\left\{a_{k}^{*}(f)\right\}_{k=1}^{\infty}-$ перестановка последовательности $\left\{\left|\widehat{a}_{k}(f)\right|\right\}_{k=1}^{\infty}$ в невозрастающем порядке и

$$
f^{*}(x)=\frac{1}{2} \widehat{a}_{0}(f)+\sum_{k=1}^{\infty} a_{k}^{*}(f) \cos (k x),
$$

mo $f^{*} \notin L_{2 \pi}$. 
ДоКАЗАТЕЛЬСТво. Выберем произвольные последовательности натуральных чисел $\left\{N_{n}\right\}_{n=1}^{\infty}$ и $\left\{K_{n}\right\}_{n=1}^{\infty}$ такие, что $2^{-n} \ln N_{n} \rightarrow+\infty$ при $n \rightarrow \infty$ и $K_{n+1}>\frac{1}{2}\left(3^{N_{n}}-1\right) K_{n}$ при $n \geqslant 1$. Пусть

$$
f(x)=\sum_{n=1}^{\infty} 2^{-n} \prod_{j=0}^{N_{n}-1}\left(1+\cos \left(3^{j} K_{n} x\right)\right) .
$$

Тогда из известных свойств произведений М. Рисса (см. [5]) сразу вытекает, что функция $f$ неотрицательна, $f \in L_{2 \pi},\|f\|_{1}=1$ и функция (6) представима в виде

$$
f^{*}(x)=1+\sum_{k=1}^{\infty} a_{k}^{*} \cos (k x)=1+\sum_{n=1}^{\infty} 2^{-n} \sum_{k=m_{n-1}}^{m_{n}-1} \cos (k x),
$$

где последовательность натуральных чисел $\left\{m_{j}\right\}_{j=0}^{\infty}$ такова, что $m_{n}-m_{n-1} \geqslant N_{n}$ при всех $n \geqslant 1$.

Для каждого натурального $n$ ряд

$$
\sin \left(m_{n} x\right)+\sum_{k=1}^{\infty} \sin \left(m_{n} x\right) a_{k}^{*} \cos (k x)
$$

сходится равномерно к функции $\sin \left(m_{n} x\right) f^{*}(x)$, поэтому

$$
\begin{gathered}
A \int_{-\pi}^{\pi}\left|f^{*}(x)\right| \cdot\left|\sin \left(m_{n} x\right)\right| d x \geqslant \frac{1}{\pi} \int_{-\pi}^{\pi} f^{*}(x) 2 \sin \left(m_{n} x\right)\left(\sum_{j=1}^{m_{n}-1} \frac{\sin (j x)}{j}\right) d x \\
\quad=\sum_{j=1}^{m_{n}-1} \frac{a_{m_{n}-j}^{*}}{j}-\sum_{j=1}^{m_{n}-1} \frac{a_{m_{n}+j}^{*}}{j} \geqslant \frac{1}{2} \sum_{j=1}^{m_{n}-1} \frac{a_{m_{n}-j}^{*}}{j} \geqslant 2^{-n-1} \sum_{j=1}^{m_{n}-1} \frac{1}{j} \\
\geqslant B 2^{-n} \ln m_{n} \geqslant B 2^{-n} \ln N_{n} \rightarrow+\infty \quad \text { при } n \rightarrow+\infty .
\end{gathered}
$$

Отсюда немедленно вытекает, что $f^{*} \notin L_{2 \pi}$.

Из рассуждений, приведенньх в работе С.А. Пичугова [6], вытекает, что для любого положительного числа $\nu$ можно указать положительное число $C_{\nu}$ такое, что для любых различных натуральных чисел $k_{1}, \ldots, k_{n}$ имеет место оценка

$$
-\min _{x} \sum_{j=1}^{n}\left(\cos \left(k_{j} x\right)-\sin \left(k_{j} x\right)\right) \geqslant C_{\nu}(\ln n)^{\nu} .
$$

Далее нам потребуется следующая

Лемма. Пусть $\Phi(z)$ - аналитическая в круге $|z|<1$ функция такая, что $\operatorname{Re} \Phi(z) \geqslant 0, \operatorname{Im} \Phi(z) \geqslant 0 n p u|z|<1, u \Phi(z)=\sum_{j=0}^{\infty} c_{j} z^{j}-$ ее разложсение в ряд Тейлора. Тогда при всех $p \in(1,2)$ функиия $\Phi \in H^{p}$ и справедливы неравенства

$$
\begin{gathered}
\|\Phi\|_{H^{p}} \leqslant\left(\frac{2}{2-p}\right)^{1 / p}|\Phi(0)|, \\
\left(\sum_{j=0}^{\infty}\left|c_{j}\right|^{q}\right)^{1 / q} \leqslant A_{5}(2-p)^{-1 / 2}|\Phi(0)|, \\
\left(\sum_{j=1}^{\infty} j^{p-2}\left(c_{j}^{*}\right)^{p}\right)^{1 / p} \leqslant A_{6}(2-p)^{-1 / 2}|\Phi(0)|,
\end{gathered}
$$

где $q=p /(p-1), a\left\{c_{j}^{*}\right\}_{j=1}^{\infty}-$ перестановка последовательности $\left\{\left|c_{j}\right|\right\}_{j=0}^{\infty}$ в невозрастающем порядке. 
ДоКАЗАТЕЛЬСтво. Возьмем произвольное положительное $\varepsilon$ и положим $G(z)=$ $\varepsilon(1+i)+\Phi(z)$ при $|z|<1$. Выберем аналитическую в круге $|z|<1$ ветвь функции $\ln G(z)=\ln |G(z)|+i \varphi(z)$, где $\varphi(z) \in(0, \pi / 2)$. При каждом $p \in[1,2)$ для всех $r \in[0,1)$ по теореме Коши имеем

$$
\frac{1}{2 \pi} \int_{0}^{2 \pi} \exp \left(p \ln G\left(r e^{i t}\right)-\frac{i \pi p}{4}\right) d t=\exp \left(p G(0)-\frac{i \pi p}{4}\right) .
$$

Приравнивая действительные части, приходим к равенству

$$
\frac{1}{2 \pi} \int_{0}^{2 \pi}\left|G\left(r e^{i t}\right)\right|^{p} \cos \left(p \varphi\left(r e^{i t}\right)-\frac{\pi p}{4}\right) d t=|G(0)|^{p} \cos \left(p \varphi(0)-\frac{\pi p}{4}\right)
$$

из которого, поскольку $|p \varphi(z)-\pi p / 4|<\pi p / 4$ при $|z|<1$, получаем неравенство

$$
\cos \left(\frac{\pi p}{4}\right) \frac{1}{2 \pi} \int_{0}^{2 \pi}\left|G\left(r e^{i t}\right)\right|^{p} d t \leqslant|G(0)|^{p} .
$$

При $\varepsilon \rightarrow 0$ получаем неравенство

$$
\frac{1}{2 \pi} \int_{0}^{2 \pi}\left|\Phi\left(r e^{i t}\right)\right|^{p} d t \leqslant \frac{|\Phi(0)|^{p}}{\cos (\pi p / 4)}
$$

которое вьполнено при всех $r \in[0,1)$. Следовательно, функция $\Phi \in H^{p}$ и справедлива оценка $\|\Phi\|_{H^{p}} \leqslant|\Phi(0)|(\cos (\pi p / 4))^{-1 / p}$. Поскольку $\cos (\pi p / 4)=\sin (\pi(2-p) / 4)>(2-p) / 2$, неравенство (7) доказано при всех $p \in[1,2)$.

Пусть далее $p \in(1,2)$. В силу известной теоремы Хаусдорфа-Юнга (см. [2, т. 2, c. 153])

$$
\left(\sum_{j=0}^{\infty}\left|c_{j}\right|^{q}\right)^{1 / q} \leqslant\|\Phi\|_{H^{p}}
$$

Отсюда и из (7) получаем

$$
\left(\sum_{j=0}^{\infty}\left|c_{j}\right|^{q}\right)^{1 / q} \leqslant\left(\frac{2}{2-p}\right)^{1 / p}|\Phi(0)| \leqslant A_{5}(2-p)^{-1 / 2}|\Phi(0)|,
$$

где

$$
A_{5}=\sup _{p \in(1,2)} \exp \left(\ln \frac{2}{p}-\frac{2-p}{2 p} \ln (2-p)\right)<2 e^{1 /(2 e)}<2 \sqrt{2} .
$$

Неравенство (8) доказано.

Из теоремы Пэли (см. [2, т. 2, с. 182-185]) имеем

$$
\left(\sum_{j=1}^{\infty} j^{p-2}\left(c_{j}^{*}\right)^{p}\right)^{1 / p} \leqslant \frac{A}{p-1}\|\Phi\|_{H^{p}}
$$

Отсюда и из (7), выполняя приведенные оценки, получаем (9) при $p \in[3 / 2,2)$. Заметим, что величина

$$
\left(6 \pi^{-2} \sum_{j=1}^{\infty} j^{-2}\left(j c_{j}^{*}\right)^{p}\right)^{1 / p}
$$

не убывает при $p \in(0,2)$. Следовательно, для некоторой постоянной $A_{6}$ неравенство $(9)$ верно при всех $p \in[1,2)$.

Отметим, что неравенство (7) известно без явного указания постоянной при $|\Phi(0)|$ (см. [2, т. 1, с. 466, задача 1]). 
ТЕорема 3. Пусть действительная функиия $f \in L_{2 \pi}$, ее сопряженная $\tilde{f} \in L_{2 \pi}$, $\int_{0}^{2 \pi} f(x) d x=0, \quad-m_{f}=\underset{x}{\operatorname{essinf}} f(x)>-\infty, \quad-m_{\tilde{f}}=\underset{x}{\operatorname{essinf}} \tilde{f}(x)>-\infty$.

Тогда при всех $p \in(1,2)$ функции $f, \tilde{f} \in L_{2 \pi}^{p}$ и справедливь неравенства

$$
\begin{gathered}
\left(\sum_{k=-\infty}^{k=+\infty}\left|\widehat{c}_{k}(f)\right|^{q}\right)^{1 / q} \leqslant A_{7}(2-p)^{-1 / 2}\left(m_{f}+m_{\tilde{f}}\right), \\
\left(\sum_{k=1}^{\infty} k^{p-2}\left(c_{k}^{*}(f)\right)^{p}\right)^{1 / p} \leqslant A_{8}(2-p)^{-1 / 2}\left(m_{f}+m_{\tilde{f}}\right),
\end{gathered}
$$

əде $q=p /(p-1)$,

$$
\widehat{c}_{k}(f)=\frac{1}{2 \pi} \int_{0}^{2 \pi} f(t) e^{-i k t} d t
$$

- комплексные коэффичиенты Фурье функции $f, a\left\{c_{k}^{*}(f)\right\}_{k=1}^{\infty}-$ перестановка последовательности $\left\{\left|\widehat{c}_{k}(f)\right|\right\}_{k=-\infty}^{\infty}$ в невозрастающем порядке.

ДоКАЗАТЕЛЬСТВО. Пусть вьполнены условия (10) и

$$
\Phi(z)=m_{f}+i m_{\tilde{f}}+\sum_{j=1}^{\infty} 2 \widehat{c}_{j}(f) z^{j} .
$$

Поскольку при $r \in[0,1)$ действительная и мнимая части функции $\Phi\left(r e^{i x}\right)$ являются абелевыми средними для неотрицательных функций $m_{f}+f(x)$ и $m_{\tilde{f}}+\widetilde{f}(x)$ соответственно, то $\operatorname{Re} \Phi(z) \geqslant 0$ и $\operatorname{Im} \Phi(z) \geqslant 0$ при $|z|<1$. Таким образом, все условия леммы выполнены и из (8) получаем

$$
\left(\sum_{n=-\infty}^{+\infty}\left|\widehat{c}_{n}(f)\right|^{q}\right)^{1 / q}=2^{-1 / p}\left(\sum_{n=1}^{\infty}\left|2 \widehat{c}_{n}(f)\right|^{q}\right)^{1 / q} \leqslant 2^{-1 / p} A_{5}(2-p)^{-1 / 2}|\Phi(0)| .
$$

Так как $|\Phi(0)|=\left|m_{f}+i m_{\tilde{f}}\right| \leqslant m_{f}+m_{\tilde{f}}$, неравенство (11) справедливо с постоянной $A_{7}=2^{-1 / 2} A_{5}<2$. Аналогично, из (9) выводим неравенство (12) с постоянной $A_{8}=A_{6}$. Теорема 3 доказана.

Следствием теоремы 3 является

Теорема 4. Для любых различных натуральных чисел $k_{1}, \ldots, k_{n}$, произвольных действительных чисел $a_{1}, \ldots, a_{n}, \varphi_{1}, \ldots, \varphi_{n}$ верно неравенство

$$
A_{10}\left(m_{f}+m_{\tilde{f}}\right) \geqslant\left(\frac{1}{1+\ln n} \sum_{j=1}^{n} a_{j}^{2}\right)^{1 / 2}
$$

әде

$$
\begin{aligned}
& f(x)=\sum_{j=1}^{n} a_{j} \cos \left(k_{j} x+\varphi_{j}\right), \widetilde{f}(x)=\sum_{j=1}^{n} a_{j} \sin \left(k_{j} x+\varphi_{j}\right), \\
& m_{f}=-\min _{x} f(x), \quad m_{\tilde{f}}=-\min _{x} \tilde{f}(x) .
\end{aligned}
$$

Если в теореме 4 положить все $\varphi_{j}=\pi / 4$, то $\widetilde{f}(x)=f(-x), m_{\tilde{f}}=m_{f}$, и из (13) сразу вытекает 
СлЕДСТВИЕ 3. Для любых различных натуральных чисел $k_{1}, \ldots, k_{n}$ и произвольных действительных чисел $a_{1}, \ldots, a_{n}$ справедливо неравенство

$$
\sqrt{2} A_{10}\left(-\min _{x} \sum_{j=1}^{n} a_{j}\left(\cos \left(k_{j} x\right)-\sin \left(k_{j} x\right)\right)\right) \geqslant\left(\frac{1}{1+\ln n} \sum_{j=1}^{n} a_{j}^{2}\right)^{1 / 2} .
$$

В частности, если все $\left|a_{j}\right| \geqslant 1$, то

$$
-\min _{x} \sum_{j=1}^{n} a_{j}\left(\cos \left(k_{j} x\right)-\sin \left(k_{j} x\right)\right) \geqslant B_{2}\left(\frac{n}{1+\ln n}\right)^{1 / 2},
$$

где $B_{2}=1 /\left(\sqrt{2} A_{10}\right)$.

Отметим, что множитель $(1+\ln n)^{-1 / 2}$ в $(15)$, а значит и в $(13)$, точен по порядку, т.e.

$$
\inf \left(-\min _{x} \sum_{j=1}^{n} a_{j}\left(\cos \left(k_{j} x\right)-\sin \left(k_{j} x\right)\right)\right) \asymp(1+\ln n)^{-1 / 2} \quad \text { при } \quad n \geqslant 1
$$

где нижняя грань берется по всем действительным числам $a_{1}, \ldots, a_{n}, \sum_{j=1}^{n} a_{j}^{2}=1$, и всем наборам различных натуральных чисел $k_{1}, \ldots, k_{n}$. Соотношение (16) вытекает из неравенства (15) и следующего факта: для каждого натурального числа $n$ можно построить не равные одновременно 0 действительные числа $a_{1}, \ldots, a_{n}$ такие, что

$$
-\min _{x} \sum_{j=1}^{n} a_{j}(\cos (j x)-\sin (j x)) \leqslant A_{11}\left(\frac{1}{1+\ln n} \sum_{j=1}^{n} a_{j}^{2}\right)^{1 / 2} .
$$

Действительно, $2 \pi$-периодическая неотрицательная функция $g$ такая, что $g(x)=$ $\sin (x / 4)(\sin (x / 2))^{-1 / 2}$ при $x \in(0,2 \pi)$, разлагается (см. [3, с. 241]) в ряд Фурье

$$
\sum_{j=0}^{\infty} b_{j}(\cos (j x)-\sin (j x)), \quad \text { где } \quad b_{j}=2^{-2 j} \frac{(2 j) !}{(j !)^{2}}, \quad j \geqslant 0
$$

Поэтому средние Фейера

$$
\sigma_{n}(g ; x)=\sum_{j=0}^{n}\left(1-\frac{j}{n+1}\right) b_{j}(\cos (j x)-\sin (j x))
$$

неотрицательны. Отсюда, полагая $a_{j}=(1-j /(n+1)) b_{j}$ при $j=1, \ldots, n$, получаем, что левая часть (17) не превышает $b_{0}=1$, a $\sum_{j=1}^{n} a_{j}^{2} \geqslant B(1+\ln n)$, поскольку $b_{j}^{2} \asymp 1 / j$ при $j \geqslant 1$ (см. [3, с. 240]). 
ДОКАЗАТЕЛЬСТВО ТЕОРЕМЫ 4. Для фуНкций (14) вьПолнены условия (10). По теореме 3 при всех $p \in(1,2)$ верно неравенство $(11)$, где $q=p /(p-1)$. Отсюда в силу неравенства Гёльдера

$$
\begin{aligned}
\sum_{j=1}^{n} a_{j}^{2} & \leqslant n^{1-2 / q}\left(\sum_{j=1}^{n}\left|a_{j}\right|^{q}\right)^{2 / q}=n^{2 / p-1}\left(\frac{1}{2} \sum_{k=-\infty}^{+\infty}\left|2 \widehat{c}_{k}(f)\right|^{q}\right)^{2 / q} \\
& \leqslant 2^{2(q-1) / q} n^{2 / p-1} A_{7}^{2}(2-p)^{-1}\left(m_{f}+m_{\tilde{f}}\right)^{2} .
\end{aligned}
$$

Следовательно,

$$
\left(\sum_{j=1}^{n} a_{j}^{2}\right)^{1 / 2} \leqslant A_{7} 2^{1 / p} n^{1 / p-1 / 2}(2-p)^{-1 / 2}\left(m_{f}+m_{\tilde{f}}\right)
$$

при всех $p \in(1,2)$. Пусть $1 / p=1 / 2+1 /(4(1+\ln n))$. Тогда

$$
\begin{aligned}
(2 n)^{1 / p-1 / 2}\left(\frac{1 / p}{2 / p-1}\right)^{1 / 2} & =\exp \left(\frac{\ln 2+\ln n}{4(1+\ln n)}\right)\left(\frac{2(1+\ln n)}{p}\right)^{1 / 2} \\
& <e^{1 / 4}(2(1+\ln n))^{1 / 2}
\end{aligned}
$$

Тем самьм,

$$
\left(\sum_{j=1}^{n} a_{j}^{2}\right)^{1 / 2} \leqslant 2 A_{7} e^{1 / 4}(1+\ln n)^{1 / 2}\left(m_{f}+m_{\tilde{f}}\right),
$$

т.е. неравенство (13) с постоянной $A_{10}=2 A_{7} e^{1 / 4}<4 e^{1 / 4}<4 \sqrt{2}<6$. Теорема 4 , а значит, и следствие 3 доказаны, причем постоянная $B_{2}=1 /\left(\sqrt{2} A_{10}\right)>1 / 8$.

Автор выражает благодарность С. В. Конягину за поддержку в работе над статьей.

\section{СПИСОК ЦИТИРОВАННОЙ ЛИТЕРАТУРЫ}

[1] Pichorides S. K. A remark on exponential sums // Bull. Amer. Math. Soc. 1977. V. 83. P. 283-285.

[2] Зигмунд А. Тригонометрические ряды. Т. 1, 2. М.: Мир, 1965.

[3] Бари Н. К. Тригонометрические ряды. М.: Физматгиз, 1961.

[4] Кусис П. Введение в теорию пространств $H^{p}$. М.: Мир, 1984.

[5] Конягин С. В. Об одной задаче Литлвуда // Матем. заметки. 1991. Т. 49. № 2. С. 143-144.

[6] Пичугов С. А. Оценки минимума тригонометрических сумм // Сб. научн. тр. "Исследования по современным проблемам суммирования и приближения функций и их приложениям". Днепропетровск: ДГУ, 1982. С. 35-38. 